\title{
Article \\ Gender and Social Inequalities in Awareness of Coronary Artery Disease in European Countries
}

\author{
Antonio Daponte-Codina 1,2 (D), Emily C. Knox ${ }^{2} \mathbb{D}$, Inmaculada Mateo-Rodriguez ${ }^{1,2,3, *(\mathbb{D})}$, Amanda Seims 4 (D), \\ Vera Regitz-Zagrosek 5,6,7, Angela H. E. M. Maas ${ }^{8}$ D, Alan White ${ }^{9}$, Floris Barnhoorn 10 \\ and Fernando Rosell-Ortiz ${ }^{11}$
}

Citation: Daponte-Codina, A.; Knox, E.C.; Mateo-Rodriguez, I.; Seims, A.; Regitz-Zagrosek, V.; Maas, A.H.E.M.; White, A.; Barnhoorn, F.; Rosell-Ortiz, F. Gender and Social Inequalities in Awareness of Coronary Artery Disease in European Countries. Int. J. Environ. Res. Public Health 2022, 19, 1388. https://doi.org/10.3390/ ijerph19031388

Academic Editors: Paul

B. Tchounwou and Stuart Gilmour

Received: 1 December 2021

Accepted: 20 January 2022

Published: 26 January 2022

Publisher's Note: MDPI stays neutral with regard to jurisdictional claims in published maps and institutional affiliations.

Copyright: (C) 2022 by the authors. Licensee MDPI, Basel, Switzerland. This article is an open access article distributed under the terms and conditions of the Creative Commons Attribution (CC BY) license (https:// creativecommons.org/licenses/by/ $4.0 /)$.
1 Andalusian School of Public Health, 18011 Granada, Spain; antonio.daponte.easp@juntadeandalucia.es 2 CIBER Epidemiology and Public Health (CIBERESP), 28029 Madrid, Spain; emily.knox.easp@juntadeandalucia.es

3 Department of Social and Organizational Psychology, Faculty of Psychology, Universidad Nacional de Educación a Distancia (UNED), 28040 Madrid, Spain

4 Bradford Teaching Hospitals NHS Foundation Trust, Bradford BD9 6RJ, UK; amanda.seims@bthft.nhs.uk

5 Department of Cardiology, University of Zurich, CH-8006 Zurich, Switzerland; vera.regitz-zagrosek@charite.de

6 Charité, Universitätsmedizin Berlin, 10117 Berlin, Germany

7 Institute for Gender Medicine, German Centre for Cardiovascular Research, Partner Site Berlin, 10785 Berlin, Germany

8 Department of Cardiology, Radboud University Medical Center, 6500 HB Nijmegen, The Netherlands; Angela.Maas@radboudumc.nl

9 School of Health and Community Studies, Leeds Beckett University, Leeds LS6 3QS, UK; alan@alanwhitemenshealth.com

10 European Public Health Association (EUPHA), 3500 BN Utrecht, The Netherlands; f.barnhoorn@euphaoffice.org

11 Medical Emergency Services 061, 26580 La Rioja, Spain; fernandorosell@gmail.com

* Correspondence: inmaculada.mateo.easp@juntadeandalucia.es; Tel.: +34-670942085

\begin{abstract}
Coronary artery disease (CAD) is the single leading cause of death in Europe and the most common form of cardiovascular disease. Little is known about awareness in the European population. A cross-sectional telephone survey of 2609 individuals from six European countries was conducted to gather information on perceptions of CAD, risk factors, preventive measures, knowledge of heart attack symptoms and ability to seek emergency medical care. Level of awareness was compared according to gender, age, socioeconomic status (SES) and educational level. Women were approximately five times less likely than men to consider heart disease as a main health issue or leading cause of death $(\mathrm{OR}=0.224,95 \% \mathrm{CI}: 0.178-0.280, \mathrm{OR}=0.196,95 \% \mathrm{CI}$ : 0.171-0.226). Additionally, women were significantly less likely to have ever had a cardiovascular screening test $(\mathrm{OR}=0.515$, $95 \%$ CI: $0.459-0.578$ ). Only $16.3 \%$ of men and $15.3 \%$ of women were able to spontaneously identify the main symptoms of a heart attack. Almost half of the sample failed to state that they would call emergency services in case of a cardiac event. Significant differences according to age, SES and education were found for many indicators amongst both men and women. Development of a European strategy targeting improved awareness of CAD and reduced gender and social inequalities within the European population is warranted.
\end{abstract}

Keywords: coronary artery disease; awareness; gender bias; sex differences; inequalities; Europe

\section{Introduction}

Coronary artery disease (CAD) accounts for $20 \%$ of all mortality in Europe and is the most common form of cardiovascular disease (CVD). According to the latest data, CAD represents $16 \%$ of all premature mortality under 75 years old among women and $18 \%$ among men [1]. Furthermore, there are large differences between countries, with 
some European countries having almost 10 times greater rates that others in both men and women [2]. These large inequalities indicate that there is great room for improvement when it comes to reducing the burden of CAD in European countries.

CAD is largely due to a limited number of risk factors, including certain behaviors [3]. It is widely recognized that improving behaviors such as smoking, following a healthy diet or engaging in physical activity can significantly reduce CHD incidence and mortality [4]. Awareness of cardiovascular disease is a hugely important factor when it comes to individuals making lifestyle changes, adopting preventive measures, and complying with health recommendations and treatment guidelines [5]. Furthermore, awareness of personal risk has been associated with the adoption of secondary prevention measures by patients, although there is a need to improve understanding of the association between health perceptions and cardiovascular preventive behaviors [6,7]. Increasing awareness is effective for improving the timely and appropriate use of different levels of health services following coronary events [8]. In short, a degree of basic knowledge about risk factors, and prevention and protection against CAD within the general population is essential for improving cardiovascular health $[9,10]$. The recent COVID-19 crisis has emphasized once more that education, socio-economic status (SES), living conditions and ethnicity are crucial factors in health and this also accounts for CAD [11]. Moreover, chronic stress induced by air pollution and traffic noise are now also well recognized as important determinants of cardiovascular health [12].

It is scientifically well established that there are important differences between men and women at all stages of the CAD process, from risk factors at the prehospital phase to diagnosis, treatment, rehabilitation and outcomes $[5,13]$. Moreover, some studies have shown that women lack awareness of CAD risk factors and symptoms, the impact of CAD on their health, measures to be taken during a coronary crisis, how to request medical assistance and options regarding transportation to hospital [14,15].

Furthermore, very few studies have addressed population awareness of CAD in Europe, in contrast with the long tradition of such research seen in other countries [10]. For all these reasons, the GenCAD project (gender specific mechanisms in coronary artery disease) was developed. This project was aimed at improving understanding of sex and gender differences in coronary artery disease in European countries.

Within the framework of this project, we carried out a study aimed at determining levels of awareness within the European population regarding essential aspects of CHD.

\section{Materials and Methods}

A cross-sectional survey was conducted of 2609 individuals from six European countries from November 2017 to March 2018.

We conducted an extensive review of the scientific literature, searching for studies on awareness of CAD and cardiovascular disease within the general population. Based on the questionnaires of selected studies $[5,9,15]$, we developed a questionnaire which included questions about coronary heart disease, specifically, perceptions of its relevance for health, risk factor identification and lifestyle behaviors, preventive measures, knowledge of heart attack symptoms (defined as myocardial infarction or angina pectoris) and capacity to seek out appropriate emergency medical care. Data were also collected on basic demographic and socioeconomic details such as sex, age, income and educational level.

The questionnaire included mostly closed questions, with the exception of questions on the following topics: CAD as a leading health issue and leading cause of death, heart attack symptoms and first reaction to suffering a hypothetical heart attack, and main risk factors related to CVD. Questions on these areas were open-ended, allowing the spontaneous responses of participants to be collected.

The questionnaire was validated through focus groups according to a series of selected criteria in order to evaluate the appropriateness and quality of questions. The final version of the questionnaire was translated into the six languages corresponding to the participating countries. Experts in cardiology and other medical specialties, psychology, 
sociology, survey development and public health, from the institutions collaborating on the project carried out this entire process. The complete questionnaire is attached online as a supplementary file.

Respondents were aged at least 25 years old and came from Bulgaria, Croatia, Czech Republic, Germany, Spain and Sweden. These countries were selected to represent the great diversity among European Member States with regards to key indicators such as CHD epidemiology, economic level, population size, and geographic location.

An estimated sample size of 2600 individuals was calculated to give an overall margin of error of $+/-1.96 \%$ according to the assumption of maximum non-determination and with a confidence level of $95 \%$. Given the large differences in population size between the selected countries, sample distribution between countries was not proportional to their respective population sizes.

Within each country, sample distribution was representative of regional variations in population size and type (towns, cities, etc.). Selection of the final sample was performed according to gender and age quotas, specific to each locality and region. A global market research company, formerly named TNS (currently Kantar), was subcontracted to adapt the questionnaire into a telephone survey, translate it into the language of each target country and conduct interviews. Within each country, households were selected at random using random digit dialing. Calls were made to both landline and mobile phones in order to maximize representativeness. Consent to participate was verbally obtained from participant subjects, after informing them of the study objectives and procedures. If a participant refused to answer or did not know how to answer a particular question, responses were coded as "don't know" or "no answer". These participants were not excluded from the analysis.

Data were also weighted to adjust for differences in the population sizes of the six countries. In this way, data reflected the European population of individuals aged 25 years and older. Further, it was noted that intra-individual income differences were not comparable between countries, e.g., A monthly income of $1000 €$ is extremely high in Bulgaria but extremely low in Sweden. Thus, the socioeconomic variable was transformed to adjust for inter-country differences relating to income, with participants' SES being classified as 'high' or 'low' relative to the median within their country. Remaining variables were coded, where appropriate, according to the needs of analysis and presentation of results.

Response data from males and females were compared to examine differences in awareness parameters. Differences within each gender according to age, educational level and SES were also explored. Detailed results are included in Figure S1 and Tables S1-S6 as online supplementary data.

Cross-tabs were produced to identify differences in responses according to gender. The Chi-squared statistic was estimated to examine the statistical relevance of differences, with significance being set at $p<0.05$. No adjustments were made for pairwise comparisons. Finally, logistic regression models were developed to compare the most important awareness indicators according to gender, adjusting for age, SES, educational level and whether or not the participant had suffered a previous heart attack. All analyses were performed using the SPSS statistical software package version 22.0.

\section{Results}

\subsection{Participant Characteristics}

Overall, $52.2 \%$ of respondents were female, $65.7 \%$ were aged older than 45 years, 38.3\% had undertaken higher educational studies and $41.1 \%$ had a high relative SES. Participants' demographic characteristics are presented in Table 1 both overall and according to gender. Female respondents were more likely than male respondents to belong to the older age group and be divorced/separated or widowed. They were less likely to report having received higher education (35.5\% versus $41.2 \%$ ) and having a high SES (36.6\% versus $45.9 \%)$. Male respondents were more likely to have had a heart attack $(6.7 \%$ versus $4.0 \%)$ and have been diagnosed with a cardiovascular disease different to CAD $(12.0 \%$ versus $8.9 \%)$. 
Table 1. Demographic characteristics of respondents to the CAD awareness survey, according to gender.

\begin{tabular}{|c|c|c|c|c|c|c|}
\hline \multirow[b]{2}{*}{ Characteristic } & \multicolumn{2}{|c|}{ Male } & \multicolumn{2}{|c|}{ Female } & \multicolumn{2}{|c|}{ Overall } \\
\hline & $\mathrm{N}$ & $\%$ & $\mathrm{~N}$ & $\%$ & $\mathrm{~N}$ & $\%$ \\
\hline Gender & 1252 & 47.8 & 1357 & 52.2 & 2609 & 100 \\
\hline \multicolumn{7}{|l|}{ Age } \\
\hline $25-44$ years & 425 & 34.0 & 469 & 34.6 & 895 & 34.3 \\
\hline 45-64 years & 514 & $41.0 *$ & 513 & $37.8^{*}$ & 1027 & 39.4 \\
\hline 65 and over & 313 & $25.0 *$ & 374 & $27.6^{*}$ & 687 & 26.3 \\
\hline Missing & 0 & & 0 & & 0 & \\
\hline \multicolumn{7}{|l|}{ Health status } \\
\hline Very good & 261 & 20.8 & 280 & 20.6 & 541 & 20.7 \\
\hline Good & 637 & 50.9 & 686 & 50.5 & 1323 & 50.7 \\
\hline Fair & 272 & $21.8^{*}$ & 325 & $23.9 *$ & 597 & 22.9 \\
\hline Bad & 65 & $5.2 *$ & 52 & $3.8^{*}$ & 117 & 4.5 \\
\hline Very bad & 17 & 1.3 & 14 & 1.0 & 31 & 1.2 \\
\hline Missing & 0 & & 0 & & 0 & \\
\hline \multicolumn{7}{|l|}{ Marital status } \\
\hline Single & 252 & 20.2 & 170 & 12.6 & 422 & 16.2 \\
\hline Married/living together & 860 & $69.1 *$ & 894 & $66.1^{*}$ & 1754 & 67.5 \\
\hline Divorced/separated & 70 & $5.6^{* *}$ & 132 & $9.8^{* *}$ & 203 & 7.8 \\
\hline Widow & 63 & $5.1^{* *}$ & 157 & $11.6^{* *}$ & 220 & 8.5 \\
\hline Missing & 0 & & 0 & & 0 & \\
\hline \multicolumn{7}{|l|}{ Education } \\
\hline Compulsory or less & 725 & $58.8 * *$ & 860 & $64.5^{* *}$ & 1584 & 61.7 \\
\hline Higher education & 509 & $41.2 * *$ & 473 & $35.5^{* *}$ & 982 & 38.3 \\
\hline Missing & 19 & 1.4 & 24 & 1.7 & 43 & 1.6 \\
\hline \multicolumn{7}{|l|}{ Relative socioeconomic status } \\
\hline High & 521 & $45.9^{* *}$ & 435 & $36.6^{* *}$ & 956 & 41.1 \\
\hline Low & 614 & $54.1 * *$ & 752 & $63.4^{* *}$ & 1366 & 58.9 \\
\hline Missing & 117 & 9.3 & 170 & 12.5 & 287 & 11.0 \\
\hline \multicolumn{7}{|l|}{ Health insurance } \\
\hline Has health coverage & 1219 & $97.4^{*}$ & 1337 & $98.5^{*}$ & 2556 & 98.0 \\
\hline Don't know/No answer & 12 & 1.0 & 10 & 0.7 & 22 & 0.8 \\
\hline \multicolumn{7}{|l|}{ Medical history } \\
\hline Has suffered from a heart attack & 83 & $6.7^{* *}$ & 54 & $4.0 * *$ & 137 & 5.3 \\
\hline Don't know/No answer & 1 & 0.1 & 1 & 0.1 & 2 & 0.1 \\
\hline Has been diagnosed with a cardiovascular disease (other than heart attack) & 148 & $12.0^{* *}$ & 120 & $8.9^{* *}$ & 268 & 10.3 \\
\hline Don't know/No answer & 13 & 1.0 & 6 & 0.4 & 19 & 0.7 \\
\hline Close relative or friend has had a heart attack & 731 & $58.7 *$ & 843 & $62.5 *$ & 1575 & 60.7 \\
\hline Don't know/No answer & 15 & 1.2 & 14 & 1.0 & 29 & 1.1 \\
\hline $\begin{array}{l}\text { Close relative or friend has had another severe cardiovascular disease } \\
\text { (different from a heart attack: myocardial infarction or angina pectoris) }\end{array}$ & 502 & 40.9 * & 589 & $44.2 *$ & 1090 & 42.6 \\
\hline Don't know/No answer & 44 & 3.5 & 41 & 3.0 & 85 & 3.3 \\
\hline $\begin{array}{c}\text { Have you ever taken a screening test to know about your risk of being } \\
\text { affected by cardiovascular disease? }\end{array}$ & 645 & $52.0 * *$ & 472 & $35.2 * *$ & 1134 & 43.2 \\
\hline Don't know/No answer & 13 & 1.0 & 14 & 1.0 & 27 & 1.0 \\
\hline
\end{tabular}

Weighted data; ${ }^{*} p<0.05 .{ }^{* *} p<0.001$.

\subsection{Awareness of Heart Disease}

A minority of males (19.2\%) and a significantly lower percentage of females $(4.2 \%)$ considered heart disease to be a leading health issue, as shown in the results presented in Table 2. Sex differences were even greater in relation to perceptions of cardiovascular disease as a leading cause of death $(49.9 \%$ men vs. $16.5 \%$ women $)$. 
Gender inequalities were also examined in relation to SES and educational level (Table 2) and age (Tables S1-S3 of supplementary material). Perception of heart disease as a leading health issue and as a leading cause of death increased with age within both males and females. Further, greater awareness of heart disease as a leading health issue was seen amongst those with a high SES compared with those with a low SES $(22.7 \%$ vs $14.2 \%$ for males; $6.7 \%$ vs. $3.0 \%$ for females), as was greater awareness of heart disease as a leading cause of death (59.4\% vs. $44.2 \%$ for males; $19.7 \%$ vs. $15.2 \%$ for females).

These inequalities were starker within men. Differences between the two educational groups only emerged within women in relation to heart disease as a leading health issue, whilst for men such differences only emerged in relation to heart disease as a leading cause of death. Complete results on these inequalities are included as supplementary material.

\subsection{Warning Signs of a Heart Attack and Calls to Emergency Services}

Table 2 presents differences in awareness of the warning symptoms of a heart attack and participants' initial response to the hypothetical experience of one. Chest pain/discomfort was the main warning sign reported, regardless of gender. Of the atypical warning signs, females were more likely to report nausea ( $12.6 \%$ vs. $5.3 \%)$ and palpitations $(10.1 \%$ versus $8.0 \%$ ). Only a small proportion of men and women were capable of spontaneously identifying the most common symptoms of a heart attack outside of pain (dyspnea, unusual fatigue, dizziness or generalised weakness) with no significant differences according to sex (16.3\% males vs. $15.4 \%$ females). Slightly more than half of respondents would call emergency services in the event of a heart attack. Women were more likely to report this response than men $(57.6 \%$ vs. $54.4 \%)$.

Knowledge of common warning signs decreased significantly with age. This knowledge was significantly higher within those with a high SES relative to those with a low SES and within those with a high educational level relative to those with low education. This was true for both males and females. In the event of a heart attack, the percentage of those who would call emergency services decreased slightly with age. Within women, differences based on educational level and SES were significant (education: 67.2\% [high] vs 53.3\% [low], SES: $64.7 \%$ [high] vs 51.9\% [low]). Within men, no significant differences emerged according to SES whilst differences were much smaller in relation to educational level. Full results can be consulted in the supplementary material.

\subsection{Perceived Risk Factors and Heart Disease Prevention Strategies}

Table 3 presents participant perceptions of the main risk factors and preventive actions. Overall, stress was the most commonly reported risk factor (63.3\%), with no significant gender differences. However, significant gender differences were found for most of the reported risk factors. Females were more likely than males to report obesity and less likely to report smoking, not exercising, and drinking alcohol.

Of the preventive actions, the most commonly cited, regardless of gender, was engaging in physical activity $(67.0 \%)$. A number of significant gender differences were found, with females being more likely to report eating fruit and vegetables, having regular medical check-ups and engaging in hypertension control practices. In contrast, males were more likely to report maintaining a healthy weight, not smoking and better stress management.

In general, a greater proportion of those belonging to younger age groups tended to accurately report the top five risk factors and the main preventive strategies. An exception to this was found for stress, with differences not being significant. Men and women with higher SES or with a higher educational level were systematically more able to identify main risk factors such as drinking alcohol, following an unhealthy diet, smoking and not exercising.

With regards to preventive actions, a greater proportion of men and women with a high educational level or high SES reported engaging in physical activity and not smoking. In contrast, those with a low socio-economic status were more likely to report having regular medical check-ups. 
Table 2. Awareness of selected leading health issues, warning signs of a heart attack and responses to signs of a heart attack, according to gender, education and SES.

\begin{tabular}{|c|c|c|c|c|c|c|c|c|c|c|c|c|c|c|c|c|c|c|c|c|}
\hline \multirow{2}{*}{ Characteristic } & \multirow{2}{*}{\multicolumn{2}{|c|}{$\mathbf{N} \quad$ Male $\%$}} & \multirow{2}{*}{\multicolumn{2}{|c|}{ Female }} & \multicolumn{4}{|c|}{ High Education } & \multicolumn{4}{|c|}{ Low Education } & \multicolumn{4}{|c|}{ High SES } & \multicolumn{4}{|c|}{ Low SES } \\
\hline & & & & & $\mathrm{x}$ & tale \% & $\mathrm{N}$ & male & N & Tale o & $\mathrm{N}$ & le of & $N$ & Male 。 & $\mathrm{r}$ & ale & $\mathrm{T}$ & Male & F & ale o \\
\hline \multicolumn{21}{|l|}{ Leading health issue (affecting your gender) } \\
\hline Cancer in general & 250 & $22.8^{*}$ & 235 & 19.9 * & 119 & $26.4^{x x}$ & 76 & 18.2 & 131 & $20.3^{x x}$ & 159 & 21.0 & 116 & 22.3 & 71 & $16.4^{t+}$ & 133 & 21.7 & 151 & $20.1^{++}$ \\
\hline Lung cancer & 19 & $1.8^{*}$ & 1 & $0.1^{*}$ & 7 & 1.6 & 1 & 0.1 & 12 & 2.0 & 0 & 0.1 & 8 & 1.7 & 0 & 0.0 & 11 & 1.9 & 1 & 0.1 \\
\hline $\begin{array}{l}\text { Diabetes } \\
\text { Heart disease /Heart attack }\end{array}$ & $\begin{array}{l}30 \\
210\end{array}$ & $\begin{array}{l}2.8 \\
19.2 * *\end{array}$ & $\begin{array}{l}28 \\
50\end{array}$ & $\begin{array}{r}2.4 \\
4.2 * *\end{array}$ & 12 & $\begin{array}{l}2.8 \\
19.7\end{array}$ & 12 & $\begin{array}{r}2.9 \\
5.9+t\end{array}$ & 18 & 2.8 & 16 & 2.22 & ${ }_{118}^{14}$ & $\begin{array}{r}2.7 \times x \\
22.7 \times x\end{array}$ & $\begin{array}{l}13 \\
28\end{array}$ & ${ }_{6.9+}^{2.9}$ & ${ }_{87}^{16}$ & $\begin{array}{l}2.8 \times \\
14.2 \times x\end{array}$ & 15 & $\begin{array}{c}2.1 \\
30^{+1}\end{array}$ \\
\hline $\begin{array}{l}\text { Heart disease//eart attack } \\
\text { Obesity }\end{array}$ & 109 & $10.0^{*}$ & 69 & $5.8^{*}$ & $\begin{array}{r}88 \\
54\end{array}$ & $\begin{array}{l}19.7 \\
12.0 \times x\end{array}$ & ${ }^{25}$ & $\begin{array}{l}5.997 \\
68^{+1}\end{array}$ & $\begin{array}{l}122 \\
55\end{array}$ & & $\begin{array}{l}25 \\
38\end{array}$ & $\begin{array}{c}3.4^{\mathrm{ft}} \\
47^{\mathrm{tt}}\end{array}$ & 118 & $11.2 \mathrm{xx}$ & 28 & $6.7^{\mathrm{TT}}$ & 52 & 年 & 22 & $3.0^{+t}+t$ \\
\hline \multirow{2}{*}{\multicolumn{19}{|c|}{$\begin{array}{l}\text { Leading cause of death (affecting your gender) } \\
\text { Accidental death }\end{array}$}} & $\begin{array}{l}26 \\
100\end{array}$ & $\begin{array}{l}3.44^{4+7} \\
13.3\end{array}$ \\
\hline & & & & & & & & & & & & & & & & & & & & $03^{2+t}$ \\
\hline Cancer in general & 260 & $22.9 *$ & 573 & $49.1^{*}$ & 101 & 21.4 & $\begin{array}{c}3 \\
202\end{array}$ & $\begin{array}{l}0.87 \\
48.7\end{array}$ & $\begin{array}{l}22 \\
159\end{array}$ & $\begin{array}{l}3.3 .7 \\
23.7\end{array}$ & $\underset{371}{2}$ & $\begin{array}{l}0.2 \\
49.2\end{array}$ & $\begin{array}{l}16 \\
100\end{array}$ & $19.1^{x \times x}$ & $\frac{3}{218}$ & $\begin{array}{l}0.99 .1 \\
50.1\end{array}$ & $\begin{array}{l}15 \\
146\end{array}$ & $23.8^{\mathrm{xx}}$ & $\frac{2}{355}$ & $\begin{array}{l}0.3 \\
47.4\end{array}$ \\
\hline $\begin{array}{l}\text { Lung cancer } \\
\text {. }\end{array}$ & 42 & $3.7^{*}$ & 13 & $1.1^{*}$ & 6 & $1.2^{2 x}$ & 0 & $0.0^{+1+}$ & 36 & $5.2^{2 \times x}$ & 13 & $1.8^{+t}$ & 7 & $1.4^{\mathrm{xx}}$ & 1 & $0.1^{+t}$ & $\begin{array}{l}190 \\
29\end{array}$ & $4.7^{\mathrm{x} x}$ & 11 & $1.4^{+t}$ \\
\hline Heart disease/Heart attack & 565 & $49.9 * *$ & 192 & $16.5^{* *}$ & 259 & $55.5^{\mathrm{xx}}$ & 75 & 18.1 & 306 & $46.1^{x x}$ & 117 & 15.6 & 301 & $59.4^{x x}$ & 83 & $19.7^{++}$ & 264 & $44.2^{x \times}$ & 109 & $15.2^{\mathrm{ft}}$ \\
\hline Stroke & 58 & $\begin{array}{l}5.1 \\
07\end{array}$ & 56 & 4.8 & 17 & $3.8^{\mathrm{xx}}$ & 17 & 4.2 & 41 & $6.1 \times x$ & 39 & 5.3 & 18 & $\begin{array}{c}3.7 \times x \\
9.3\end{array}$ & 13 & $2.9^{t+}$ & 40 & $6.9^{\mathrm{xx}}$ & 43 & $6.0^{\mathrm{tt}}$ \\
\hline \multicolumn{21}{|c|}{$\begin{array}{l}\text { Don't know/No answer } \\
\text { What are the warning signs that you associate with having a heart attack? }\end{array}$} \\
\hline 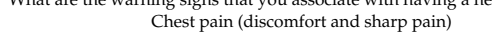 & 759 & $60.6 * *$ & 779 & $57.4^{* *}$ & 334 & $65.6^{\mathrm{xx}}$ & 305 & $64.4^{+t}$ & 418 & $57.6^{\mathrm{xx}}$ & 467 & $54.3^{\mathrm{tt}}$ & 340 & $65.3 \times x$ & 288 & 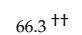 & 345 & $56.2 \times x$ & 399 & $53.0^{\mathrm{t+}}-2-0$ \\
\hline Radiation of pain & 532 & $42.5 * *$ & 659 & $48.6^{* *}$ & 221 & 43.5 & 246 & $52.0^{\dagger}$ & 303 & 41.8 & 405 & $47.1^{\dagger}$ & 237 & $45.4^{\times x}$ & 241 & $55.4^{t+}$ & 234 & $38.1^{x \times}$ & 338 & 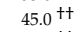 \\
\hline Dyspnea (shortness of breath) & 219 & $17.5^{*}$ & 268 & $19.7 *$ & 97 & 19.1 & 96 & 20.4 & 122 & 16.8 & 169 & 19.7 & 111 & $21.3^{x x}$ & 111 & $25.6^{t+}$ & 89 & $14.5^{\mathrm{xx}}$ & 129 & $17.2^{\mathrm{t+}}$ \\
\hline Nausea & 66 & $5.3^{* *}$ & 171 & $12.6^{* *}$ & 36 & $7.0^{x x}$ & 68 & 14.4 & 29 & $4.0^{x x}$ & 102 & 11.9 & 36 & $7.0^{x x}$ & 42 & $9.7^{\dagger}$ & 22 & $3.6^{x x}$ & 102 & $13.6^{+}$ \\
\hline Sweating & 110 & $8.8^{* *}$ & 55 & $4.1^{* *}$ & 51 & 10.1 & 22 & 4.6 & 59 & 8.1 & 31 & 3.6 & 58 & $11.1 \times x$ & 17 & 3.9 & 39 & $6.4^{\mathrm{xx}}$ & 33 & 4.4 \\
\hline Unusual fatigue & 60 & 4.8 & 64 & 4.7 & 18 & $3.6^{x}$ & 29 & $6.2^{\dagger}$ & 42 & $5.8^{\mathrm{x}}$ & 34 & $4.0^{\dagger}$ & 20 & $3.9^{x}$ & 20 & 4.7 & 36 & $5.8^{x}$ & 38 & 5.1 \\
\hline $\begin{array}{c}\text { Dizziness } \\
\text { Perlined }\end{array}$ & 180 & $14.4^{*}$ & 160 & $11.8^{*}$ & 95 & $18.6^{x x}$ & 59 & 12.4 & 85 & $11.8^{x x}$ & $\begin{array}{r}99 \\
40\end{array}-10$ & 11.5 & 77 & 14.7 & ${ }_{20}^{56}$ & 12.8 & 88 & 14.4 & 91 & 12.1 \\
\hline $\begin{array}{l}\text { Generalaized weakness } \\
\text { Palpitations }\end{array}$ & $\begin{array}{l}83 \\
100\end{array}$ & $\begin{array}{l}6.7 * \\
8.0 *\end{array}$ & $\begin{array}{l}62 \\
138\end{array}$ & $\begin{array}{l}4.6^{*} \\
10 .{ }^{*}\end{array}$ & $\begin{array}{l}38 \\
45\end{array}$ & $\begin{array}{l}7.4 \\
89\end{array}$ & $\begin{array}{l}22 \\
48\end{array}$ & $\begin{array}{l}4.7 \\
10.1\end{array}$ & $\begin{array}{l}45 \\
52\end{array}$ & $\begin{array}{l}6.3 \\
7.2\end{array}$ & $\begin{array}{l}40 \\
84\end{array}$ & $\begin{array}{l}4.7 \\
9.8\end{array}$ & $\begin{array}{l}33 \\
35\end{array}$ & $\begin{array}{l}6.3 \\
6.8 x \\
x\end{array}$ & $\frac{22}{48}$ & $\begin{array}{l}5.0 \\
11.1\end{array}$ & $\begin{array}{l}45 \\
58\end{array}$ & $\begin{array}{l}7.4 \\
9.4 \times\end{array}$ & $\begin{array}{l}39 \\
79\end{array}$ & $\begin{array}{c}5.2 \\
10.5\end{array}$ \\
\hline $\begin{array}{l}\text { Palptitatons } \\
\text { Don't know/No answer }\end{array}$ & $\begin{array}{l}100 \\
305\end{array}$ & $\begin{array}{l}8.0^{\circ} \\
24.4 \\
-10\end{array}$ & $\begin{array}{l}138 \\
140\end{array}$ & $\begin{array}{l}10.1 * \\
10.3\end{array}$ & $\begin{array}{l}45 \\
109\end{array}$ & $\begin{array}{l}8.9 \\
21.4\end{array}$ & $\begin{array}{l}48 \\
35\end{array}$ & $\begin{array}{l}10.1 \\
7.5\end{array}$ & $\begin{array}{l}52 \\
188\end{array}$ & $\begin{array}{l}7.2 \\
25.9\end{array}$ & $\begin{array}{l}84 \\
104\end{array}$ & $\begin{array}{l}9.8 \\
12.1\end{array}$ & $\begin{array}{l}30 \\
105\end{array}$ & $\begin{array}{l}6.8 \times 1 \\
20.1\end{array}$ & ${ }_{22}^{48}$ & 5.1 & $\begin{array}{l}58 \\
174\end{array}$ & $\begin{array}{l}9.4 \times \\
28.4\end{array}$ & $\begin{array}{l}79 \\
97\end{array}$ & $\begin{array}{l}10.5 \\
12.9\end{array}$ \\
\hline Knows the main symptoms & \multicolumn{18}{|c|}{$\begin{array}{l}\text { Knows the main symptoms } \\
\text { If you thought you were experiencing a hart attack, what is the }\end{array}$} & & \\
\hline Call a doctor & 187 & $15.0^{*}$ & 227 & $16.7^{*}$ & 68 & $13.4^{x \times}$ & 51 & 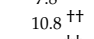 & 117 & $16.1^{x x}$ & 166 & $19.3^{+t}$ & 77 & 14.8 & 59 & $13.5^{\mathrm{tt}}$ & 91 & 14.9 & 149 & 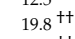 \\
\hline Call emergency medical services (112) & 681 & $54.4^{*}$ & 781 & $57.6 *$ & 298 & $58.6^{\mathrm{xx}}$ & 317 & $67.1^{++}$ & 376 & $51.8^{\mathrm{xx}}$ & 458 & $53.3^{+t}$ & 286 & 54.8 & 281 & $64.7^{+t}$ & 327 & 53.3 & 390 & 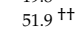 \\
\hline Call your spouse or a family member & 109 & $8.7^{*}$ & & $6.9^{*}$ & 51 & $10.1^{x x}$ & 36 & 7.6 & 57 & $7.9^{x x}$ & & & & & & & & & & \\
\hline $\begin{array}{l}\text { Oall your spouse or alminy memier } \\
\text { Other }\end{array}$ & 119 & $9.5^{*}$ & ${ }_{71}^{94}$ & $5.2^{*}$ & 43 & 8.5 & 21 & 4.5 & 68 & 9.4 & $\begin{array}{l}56 \\
50\end{array}$ & 5.8 & $\begin{array}{l}49 \\
60\end{array}$ & $11.6 \times x$ & 24 & 5.5 & $\begin{array}{l}52 \\
47\end{array}$ & $7.7^{8 \times x}$ & 38 & $\begin{array}{l}0.3 .0 \\
5.0\end{array}$ \\
\hline Don't know & 28 & 2.2 & 33 & 2.5 & 3 & $0.5^{\mathrm{xx}}$ & 4 & $0.8^{\mathrm{tt}}$ & 25 & $3.4^{\mathrm{xx}}$ & 28 & $3.3^{t+}$ & 4 & $0.7^{x \times}$ & 4 & $1.0^{\mathrm{tt}}$ & 23 & $3.7^{x x}$ & 24 & $3.2^{t+}$ \\
\hline Call emergency services versus all other options & 681 & $54.4^{*}$ & 781 & $57.6^{*}$ & 299 & $58.7^{x x}$ & 318 & $67.2^{+t}$ & 376 & $51.8^{\mathrm{xx}}$ & 458 & $53.3^{+t}+2>0$ & 286 & 54.8 & 281 & $64.7^{+t}$ & 327 & 53.3 & 390 & $51.9^{+t}$ \\
\hline
\end{tabular}


Table 3. Main risk factors, preventive actions and individuals most at risk of cardiovascular disease, according to gender, education and SES

\begin{tabular}{|c|c|c|c|c|c|c|c|c|c|c|c|c|c|c|c|c|c|c|c|c|}
\hline \multirow[b]{2}{*}{ Characteristic } & \multirow{2}{*}{$\mathrm{N}$} & \multirow[b]{2}{*}{ Male $\%$} & \multirow{2}{*}{\multicolumn{2}{|c|}{$\mathrm{N}$ Female $\%$}} & \multicolumn{4}{|c|}{ High Education } & \multicolumn{4}{|c|}{ Low Education } & \multicolumn{4}{|c|}{ High SES } & \multicolumn{4}{|c|}{ Low SES } \\
\hline & & & & & $\mathbf{N}$ & Male $\%$ & $\mathrm{~N}$ & ale $\%$ & $\mathbf{N}$ & Male $\%$ & $N$ & ale $\%$ & $\mathrm{~N}$ & Male $\%$ & $N$ & ale $\%$ & $N$ & $\begin{array}{ll}\text { ale } & \%\end{array}$ & $N$ & nale \\
\hline Main risk factors for suffering cardiovascular disease & & & & & & & & & & & & & & & & & & & & \\
\hline High blood pressure & 142 & $11.4^{* *}$ & 251 & $18.5 * *$ & 81 & $16.0^{x x}$ & 109 & $23.0^{t+}$ & 60 & $8.3^{x x}$ & 141 & $16.4^{\mathrm{t+}}$ & 67 & 18.8 & 58 & $13.4^{++}$ & 75 & 17.8 & 65 & $8.7^{\text {t+ }}$ \\
\hline Cholesterol (low good cholesterol or high bad cholesterol) & 74 & $5.9 * *$ & 142 & 10.4 ** & 46 & $9.1 \times x$ & 64 & $13.5^{++}$ & 28 & $3.9 \times x$ & 74 & $8.6^{\mathrm{t+}}$ & 37 & 11.0 & 32 & $7.4^{+t}$ & 37 & 9.4 & 29 & $3.8^{t+}$ \\
\hline Family history of heart disease or stroke & 128 & $10.2^{* * *}$ & 211 & 15.6 ** & 65 & $12.8^{\mathrm{xx}}$ & 108 & $22.8^{++}$ & 62 & $8.6^{6 x}$ & 97 & $11.3^{t+}$ & 69 & $18.7^{\mathrm{x}}$ & 50 & $11.6^{\dagger}$ & 59 & $13.4^{x}$ & 64 & $8.5^{\dagger}$ \\
\hline Smoking habit & 794 & $63.4^{*}$ & 812 & $59.8^{*}$ & 322 & 63.2 & 300 & $63.4^{+}$ & 455 & 62.7 & 499 & $58.0^{+}$ & 369 & $60.4^{x}$ & 304 & $69.8^{\mathrm{tt}}$ & 351 & $48.9 \times x$ & 429 & $57.0^{\mathrm{t}}$ \\
\hline Drinking alcohol & 598 & $47.8^{* *}$ & 376 & 27.7 ** & 231 & 45.4 & 134 & 28.3 & 354 & 48.8 & 237 & 27.5 & 258 & $37.0^{x}$ & 147 & $50.6^{\dagger}$ & 262 & $32.0^{x}$ & 229 & $45.3^{\dagger}$ \\
\hline Diabetes & 46 & $3.6^{* *}$ & 99 & $7.3^{* *}$ & 29 & $5.8 \times x$ & 41 & $8.7^{\dagger}$ & 17 & $2.3^{x x}$ & 58 & $6.7^{\dagger}$ & 22 & 6.3 & 20 & $4.5^{\dagger}$ & 24 & 5.7 & 21 & $3.0^{\dagger}$ \\
\hline Unhealthy diet habits & 489 & 39.1 & 544 & 40.1 & 223 & $\begin{array}{l}43.8^{8 x} \\
-1-x x\end{array}$ & 225 & $47.6^{t+}$ & 259 & $35.7^{x x}$ & 311 & $36.2^{++}$ & 230 & $44.2^{x}$ & 181 & $41.5^{\dagger}$ & 221 & $36.0^{x x}$ & 272 & $36.2^{\dagger}-x-2$ \\
\hline Not exercising & 537 & $42.9^{*}$ & 542 & $40.0^{*}$ & 277 & $54.5^{x x}$ & 236 & $49.9^{+t}$ & 258 & $35.6^{6 x}$ & 296 & $34.4^{++}$ & 254 & $48.7^{x}$ & 226 & $51.9^{t+}$ & 200 & $32.5^{x x}$ & 262 & $34.9^{+t}$ \\
\hline Obesity & 394 & $31.4 * *$ & 501 & $36.9^{* *}$ & 182 & $35.8^{x x}$ & 187 & $39.6^{\dagger}$ & 210 & $28.9^{x x}$ & 296 & $34.4^{\dagger}$ & 207 & $39.8^{x}$ & 187 & $43.1^{\mathrm{t+}}$ & 153 & $24.9^{x x}$ & 162 & $21.6^{\mathrm{t}}$ \\
\hline $\begin{array}{l}\text { Stress } \\
\text { Onther }\end{array}$ & 804 & 64.2 & 847 & 62.4 & 313 & $61.4^{x}$ & 313 & $66.2^{\dagger}$ & 473 & $65.3^{x}$ & 529 & $61.5^{+}$ & 353 & 67.7 & 288 & 66.3 & 403 & 65.6 & 475 & 63.1 \\
\hline $\begin{array}{l}\text { Other } \\
\text { Don't know }\end{array}$ & $\begin{array}{l}95 \\
143\end{array}$ & $\begin{array}{l}7.6^{* *} \\
114\end{array}$ & $\begin{array}{l}226 \\
72\end{array}$ & $\begin{array}{l}16.7 * * \\
53\end{array}$ & $\begin{array}{l}35 \\
40\end{array}$ & $\begin{array}{l}6.9 \\
78\end{array}$ & $\begin{array}{l}89 \\
14\end{array}$ & $18.9^{\dagger}$ & $\begin{array}{l}59 \\
103\end{array}$ & $\begin{array}{l}8.2 \\
142\end{array}$ & 134 & $15.6^{\dagger}$ & 35 & $10.2^{x}$ & 51 & 11.7 & 60 & $15.0^{x}$ & 105 & 13.9 \\
\hline Preventive actions & & 11.4 & 72 & 5.3 & 40 & 7.8 & 14 & & 103 & 14.2 & 52 & & 30 & 5.8 & 7 & 1.7 & 103 & 16.8 & 47 & 6.2 \\
\hline Eating more fruit and vegetables & 544 & $43.4^{*}$ & 626 & $46.3 *$ & 211 & 41.5 & 178 & $37.6^{t+}$ & 324 & 44.7 & 437 & $50.8^{\dagger+}$ & 212 & 40.6 & 201 & 46.1 & 273 & 44.4 & 365 & 48.6 \\
\hline Physical activity & 846 & 67.6 & 891 & 65.9 & 362 & $71.2^{\mathrm{x}}$ & 339 & $71.6^{t+}$ & 481 & $66.3^{x}$ & 543 & 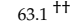 & 389 & $74.7 \times x$ & 309 & $71.0^{++}$ & 395 & $64.4^{\times x}$ & 483 & 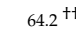 \\
\hline Regular medical check-ups & 351 & $28.1^{*}$ & 409 & $30.3 *$ & 126 & $24.8^{x}$ & 152 & 32.1 & 220 & $30.3^{x}$ & 249 & 29.0 & 135 & $25.9^{x}$ & 120 & $27.5^{+}$ & 191 & $31.1^{x}$ & 237 & $31.5^{+}-100$ \\
\hline Keep a healthy weight & 472 & $37.7 *$ & 478 & $35.3^{*}$ & 215 & $42.2^{x \times}$ & 187 & $39.5^{\mathrm{t+}}$ & 247 & $34.0^{x \times}$ & 278 & $32.3^{t+}$ & 212 & $40.7^{x}$ & 161 & 37.0 & 213 & $34.7^{x}$ & 255 & 33.9 \\
\hline Not smoking & 636 & $50.8^{* *}$ & 638 & $47.2^{\text {** }}$ & 269 & 2.8 & 245 & $51.8^{t+}$ & 360 & 49.6 & 378 & $44.0^{+t}$ & 276 & $53.0^{x}$ & 223 & $51.3^{t+}$ & 289 & $47.0^{x}$ & 316 & $42.0^{\mathrm{t}}$ \\
\hline Improve stress management & 334 & 26.7 ** & 321 & 23.8 ** & 145 & $28.4^{\mathrm{x}}$ & 121 & 25.5 & 180 & $24.8^{x}$ & 200 & 23.4 & 148 & 28.5 & 120 & $27.5^{t+}$ & 161 & 26.3 & 157 & $20.9^{+\mathrm{t}}-3-3-3-1$ \\
\hline Hypertension control & 275 & 22.0 ** & 374 & $27.7 * *$ & 99 & $19.4^{x}$ & 130 & 27.4 & 171 & $23.6^{x}$ & 237 & 27.6 & 106 & 20.3 & 107 & $24.7^{\dagger}$ & 136 & 22.2 & 217 & $28.8^{\dagger}-x-3-1$ \\
\hline Taking vitamins & 18 & $1.4^{*}$ & 30 & $2.2^{*}$ & 6 & 1.2 & 7 & $1.4^{+}$ & 9 & 1.3 & 23 & $2.7^{\dagger}$ & 1 & $0.2^{x x}$ & 8 & 1.8 & 14 & $2.3^{x x}$ & 22 & 2.9 \\
\hline Taking antioxidants & 12 & 0.9 & 15 & 1.1 & 4 & 0.7 & 5 & ${ }^{1.1}+{ }_{t}$ & 8 & 1.1 & 10 & 1.2 & 2 & $0.4^{x}$ & 5 & 1.1 & 9 & $1.4^{\mathrm{x}}$ & 10 & 1.3 \\
\hline $\begin{array}{l}\text { Hormone replacement therapy } \\
\text { Diaibetes sontrol }\end{array}$ & 4 & 0.3 & 3 & 0.2 & 0 & $0.0^{x}$ & 0 & $0.0^{\dagger}$ & 4 & $0.5^{\mathrm{x}}$ & 3 & $0.4^{+}$ & 1 & 0.1 & 1 & 0.1 & 2 & 0.4 & 1 & 0.1 \\
\hline $\begin{array}{l}\text { Diabetes control } \\
\text { Don't know }\end{array}$ & 117 & $9.3^{*}$ & 108 & $8.0^{*}$ & 44 & 8.6 & 28 & $6.0^{\dagger}$ & 73 & 10.0 & 77 & $8.9^{\dagger}$ & 39 & $7.5^{\mathrm{x}}$ & 21 & $4.9^{+\dagger}$ & 61 & $9.9 \times$ & 74 & $9.9^{+t}$ \\
\hline $\begin{array}{l}\text { Don't know } \\
\text { Individuals who are most at risk of suffering from carc }\end{array}$ & 13 & 1.1 & 10 & 0.7 & 4 & 0.8 & 1 & $0.2^{+}$ & 9 & 1.3 & 9 & $1.0^{\dagger}$ & 2 & $0.3^{x}$ & 3 & 0.7 & 10 & $1.7^{x}$ & 3 & 0.4 \\
\hline Men have more heart diseases than women & 960 & $80.2 * *$ & 892 & $69.9 * *$ & 417 & $82.0^{x}$ & 339 & $74.7^{\mathrm{t+}}$ & 542 & $78.8^{x}$ & 553 & $67.0^{\mathrm{t+}}$ & 443 & $85.1 \times x$ & 312 & 71.7 & 461 & $75.0^{x x}$ & 517 & 68.8 \\
\hline 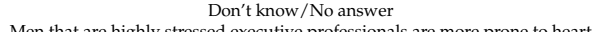 & 55 & 4.4 & 81 & 6.0 & 29 & 5.7 & 24 & 5.0 & 26 & 3.6 & 54 & 6.3 & 24 & 4.7 & 14 & 3.3 & 21 & 3.4 & 44 & 5.8 \\
\hline $\begin{array}{l}\text { Men that are highly stressed executiviv professionals are more prone to heart } \\
\text { attacks }\end{array}$ & 1059 & 85.7 & 1151 & 86.2 & 436 & 85.6 & 406 & 85.8 & 621 & 85.7 & 740 & 86.1 & 450 & 86.4 & 378 & 86.9 & 533 & 86.8 & 650 & 86.4 \\
\hline Don't know/No answer & 15 & 1.2 & 22 & 1.6 & 9 & 1.7 & 1 & 0.2 & 7 & 0.9 & 22 & 2.5 & 5 & 0.9 & 1 & 0.1 & 10 & 1.0 & 15 & 2.0 \\
\hline $\begin{array}{l}\text { Young women, under 50, do not have heart attacks } \\
\text { Don't know } / \text { No answer }\end{array}$ & 310 & $25.7^{* *}$ & 274 & $20.8 * *$ & 109 & $21.8^{\mathrm{xx}}$ & 100 & 21.3 & 201 & $28.4^{x x}$ & 174 & 20.6 & 126 & $24.1^{x}$ & 78 & $17.9^{\dagger}$ & 176 & $28.7^{x}$ & 165 & $21.9^{\dagger}$ \\
\hline $\begin{array}{l}\text { Don't know/No answer } \\
\text { In women the probability of heart disease increases after menopause }\end{array}$ & $\begin{array}{l}46 \\
667\end{array}$ & $\begin{array}{l}3.7 \\
65^{* * *}\end{array}$ & $\begin{array}{l}35 \\
830\end{array}$ & $\begin{array}{c}2.6 \\
69.1 * *\end{array}$ & 222 & $\begin{array}{r}4.3 \\
68.4^{x}\end{array}$ & $\begin{array}{c}4 \\
320\end{array}$ & $\begin{array}{c}0.9+ \\
751+1\end{array}$ & 25 & $3.5 \mathrm{x}$ & 32 & 3.7 & 18 & 3.4 & 1 & 0.1 & 23 & 3.8 & 27 & 3.6 \\
\hline $\begin{array}{l}\text { In women the probabilily of heart disease increases after menopause } \\
\text { Don't know w No answer }\end{array}$ & $\begin{array}{l}637 \\
230\end{array}$ & $\begin{array}{l}65.2^{2 * 4} \\
18.4\end{array}$ & $\begin{array}{l}830 \\
156\end{array}$ & $\begin{array}{c}69.1 \% 4 \\
11.5\end{array}$ & $\begin{array}{l}288 \\
112\end{array}$ & $\begin{array}{l}68.4 \times \\
22.0\end{array}$ & $\begin{array}{l}320 \\
50\end{array}$ & $\begin{array}{c}75.111 \\
10.6\end{array}$ & $\begin{array}{l}379 \\
112\end{array}$ & $\begin{array}{l}63.3^{x} \\
15.5\end{array}$ & $\begin{array}{l}510 \\
106\end{array}$ & $\begin{array}{c}65.811 \\
12.3\end{array}$ & 298 & $\begin{array}{l}63.1 \\
18.6\end{array}$ & $\begin{array}{l}308 \\
38\end{array}$ & $\begin{array}{l}70.7 \\
8.7\end{array}$ & 369 & $\begin{array}{c}66.3 \\
17.8\end{array}$ & $\begin{array}{l}506 \\
85\end{array}$ & $\begin{array}{l}67.3 \\
11.3\end{array}$ \\
\hline $\begin{array}{l}\text { Only women who adopt behaviors and lifestyles of men will have heart } \\
\text { disease }\end{array}$ & 290 & $24.7^{*}$ & 301 & $22.9 *$ & 99 & $20.5^{x x}$ & 64 & $13.7^{t+}$ & 191 & $27.9^{x x}$ & 237 & $27.9^{t+}$ & 122 & $23.5^{x}$ & 72 & $16.6^{t+}$ & 167 & $27.2^{x}$ & 199 & $26.5^{+}$ \\
\hline $\begin{array}{l}\text { Don't know } / \text { No answer } \\
\text { diste }\end{array}$ & 78 & 6.2 & 46 & 3.4 & 30 & 5.8 & 15 & 3.1 & 48 & 6.6 & 31 & 3.6 & 22 & 4.3 & 2 & 0.4 & 47 & 7.7 & 34 & 4.5 \\
\hline Only women which have brought up children will have heart disease & 55 & 4.6 & 54 & 4.0 & 13 & $2.6^{6 x}$ & 7 & $1.5^{\mathrm{tt}}$ & 42 & $6.1^{x \times}$ & 45 & $5.2^{++}$ & 21 & 4.0 & 7 & $1.7^{+\dagger}$ & 28 & 4.6 & 43 & $5.7^{+1}$ \\
\hline Don't know/No answer & 70 & 5.6 & 31 & 2.3 & 21 & 4.2 & 7 & 1.5 & 30 & 4.2 & 25 & 2.9 & 18 & 3.4 & 1 & 0.1 & 31 & 5.0 & 23 & 3.1 \\
\hline
\end{tabular}

${ }^{*}$ indicates overall comparisons; ${ }^{\mathrm{x}}$ indicates comparisons between male subgroups; ${ }^{+}$indicates comparisons between female subgroups. ${ }^{*}, \mathrm{x},{ }^{+} p<0.05 .{ }^{* *}, \mathrm{xx},{ }^{+\dagger} p<0.001$. Weighted data 


\subsection{At-Risk Individuals and Medical History}

Table 3 presents perceptions about the types of individuals who are most at risk of suffering from heart disease. A very high percentage of respondents, regardless of gender, agreed with the statement that "highly stressed executive professionals are more prone to heart attacks". Females were less likely than males to agree with the statements that "men have more heart disease than women" and that "probability of heart disease in women increases after menopause", and more likely to agree that "young women, under 50, do not have heart attacks".

Table 3 also presents inequalities pertaining to medical history according to SES and educational level. A significantly higher proportion of those with low SES or with a low educational level had previously had a heart attack, with differences being particularly large amongst women. Differences in having had a screening test on CHD risk were not systematic. The proportion of both men and women to have taken screening tests increased with advancing age. Within men, differences were significant when stratifying according to SES but not when stratifying according to educational level. Within women, the opposite was found with a greater proportion of screening tests being taken by those with a low SES and low educational level than those with a high SES (36.9\% vs. $32.1 \%)$ and high educational level (37.6\% vs. $32.1 \%)$, respectively.

\subsection{Key Awareness Indicators}

Table S6 of supplementary material shows the odds ratios predicting awareness of 11 key indicators in women relative to men, following adjustment for age, SES, educational level and whether one had suffered a previous heart attack. These odds ratios are presented in Figure 1. Results showed that women were approximately five times less likely to consider heart disease as the greatest health issue or leading cause of death amongst women (OR $=0.224,95 \%$ CI: $0.178-0.280$, OR $=0.196,95 \%$ CI: 0.171-0.226). Further, women were significantly less likely to have discussed risk factors with their doctors (OR $=0.460$, 95\% CI: 0.408-0.518) and have ever taken a cardiovascular screening test. No gender differences were found with regards to knowledge of the main signs of a heart attack, although women were somewhat more likely to call emergency services than men should they suffer a heart attack. Finally, women in the present sample were significantly less likely to have had a heart attack (OR $=0.536,95 \%$ CI: 0.415-0.691).

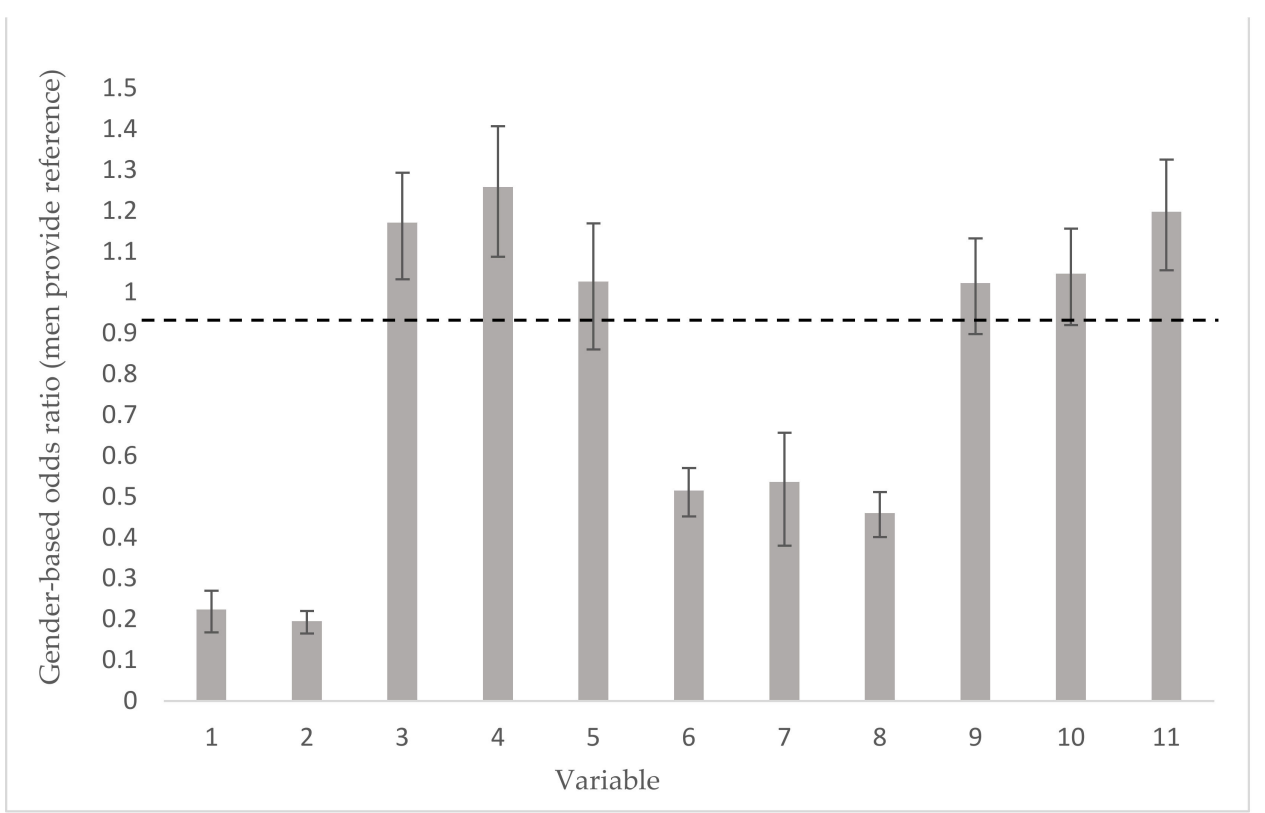

Figure 1. Odds Ratios and 95\% CIs pertaining to selected indicators of awareness. Gender odds ratios 
and associated 95\% CIs adjusted for age, SES, education and prior experience of a heart attack (males provide reference group), with the exception of variable 7 which is adjusted for age, SES and education. Dashed line is $\mathrm{OR}=1$, i.e., no gender difference. Weighted data. (1) Heart diseases is a main health issue for your gender; (2) Heart diseases is a leading cause of death for your gender; (3) Would call emergency services when suffering a heart attack; (4) Would call emergency services if somebody else suffered a heart attack; (5) Knows main warning signs of a heart attack; (6) Has taken a cardiovascular screening test ever; (7) Has suffered a heart attack; (8) Doctor has discussed risk factors; (9) Would like more information on heart disease; (10) I am informed about heart disease and the risk factors associated with it; (11) Knows what to do and how to do it, when it comes to preventing heart disease. $p<0.05$ for variables $1-4,6-8$ and 11 .

\section{Discussion}

The present study shows that levels of awareness in relation to CAD in this sample of the European population are far from adequate. Further, the study reveals that women have systematically lower awareness than men for many of the studied aspects. Whilst it is true that CVD tends to develop at a later age in women [16], it has similar epidemiological outcomes in men and women and, yet women gave it much less importance than men. This is made worse by the fact that significant social inequalities also emerged in key aspects of awareness of cardiovascular health. This was despite the fact that the studied population was familiar with heart disease, as evidenced by the high percentage reporting having personally suffered a CVD or having a family member or close friend who had.

Awareness that heart disease is a major health issue was notably low and the majority of individuals also failed to recognize it as a leading cause of death. Similarly, a study carried out in five European countries almost two decades ago found that less than half of participants were able to correctly identify CHD as the leading cause of death [17]. It seems, therefore, that no major changes in CHD awareness amongst the European general public have occurred, despite the large expansion of medical, epidemiological and preventive knowledge about this disease over the last 20 years. In the present study, awareness of CHD as an important health issue or as the leading cause of death was much lower amongst women. Women attribute much less importance to heart disease than it warrants due to the epidemiological significance of related pathologies within women. This is especially true when we consider that CVD mortality amongst women in Europe is higher than their combined mortality from all cancers [1].

It is also of interest that when comparing present results with the results of reference studies from the USA, it can be seen that the percentage of present respondents aware that CHD is the leading cause of death is well below the percentage seen amongst American women [9]. As is the case in other studies, our results show that gender inequalities are further exacerbated by inequalities according to SES, education and age [18]. Moreover, in the present study, the outcome of combining inequalities in gender and SES was alarming. For instance, the percentage of low SES women who considered CHD to be a leading health issue $(3.0 \%$ vs. $22.7 \%)$ or a leading cause of death $(15.2 \%$ vs. $59.4 \%)$ was seven and four times lower, respectively, than that of high SES men.

For the population as a whole, CAD awareness is essential for the prevention of cardiovascular disease and associated mortality. The fact that women give greater relevance to other health problems with much less epidemiological significance suggests that gender specific effective strategies are needed $[5,18]$.

Pain is the most cited symptom of a heart attack, being reported by just over half of those surveyed. This result is similar to that produced in other studies [18-22]. Although respondents were less likely to identify other typical heart attack symptoms, as was the case in other studied populations, they did identify the most important ones [17,22-24]. Exploring this further, women identified some of the atypical symptoms somewhat more frequently than men, although differences were small. The lack of differences in relation to identified symptoms is important as it places women at a disadvantage when it comes to 
recognizing and acting in the event of a heart attack since they tend to present with atypical symptoms $[25,26]$.

In any case, spontaneous knowledge about the set of symptoms that characterize a heart attack is very low, being even lower in the present study than in studies of other European or American populations. These differences between studies may be due to the fact that the present study examined spontaneous responses, whereas other studies were based on predefined lists of symptoms [23]. Present results show that older people were less able to identify the symptoms of a heart attack. Results of previously published studies are not consistent in this regard [20,24,27]. Given that the vast majority of heart attacks occur at older ages, the consequences of this lack of knowledge could be important. In addition, correct symptom identification was poorer amongst people with a low educational level and low SES. This has also been found in other studies $[14,19,24,27,28]$. In the event of a heart attack, slightly more than half of participants would call emergency services. This percentage was somewhat higher within women and those belonging to younger age groups. Following adjustment for sociodemographic characteristics and previous heart attack history, the likelihood of calling emergency services was significantly higher amongst women. In addition, this percentage was significantly lower within men and women with a low educational level and SES [20,22,27].

Lack of knowledge of heart attack symptoms was associated with a failure to engage in the recommended action and call emergency services when suffering a heart attack [20]. Social inequalities both in the recognition of symptoms and in calling emergency services may significantly contribute to greater delays in receiving appropriate health care $[29,30]$. This could, therefore, contribute to the already known social inequalities in heart attack survival [31].

Both males and females reported stress to be the main risk factor for CHD, which is in itself relevant for cardiovascular health [32]. Lifestyle risk factors such as smoking, diet and not engaging in physical activity were more recognized than clinical factors. Signaled preventive activities reasonably correspond with prioritized risk factors and these results, in general, agree with those of previous studies and are in line with scientific evidence and with European recommendations $[10,14,16,33]$.

Furthermore, differences emerged in relation to age. Both men and women were less likely to identify lifestyle risk factors with increasing age. Findings around the underestimation of this risk with age have already been described in women. Previous studies have also described how the percentage of women who identify clinical measures as main preventive measures, such as hypertension and diabetes control, increases with age $[5,10]$.

SES and educational level are important factors when it comes to demonstrating inequality in the knowledge of risks and preventive measures [5,14,34]. Present results show that individuals with a high SES and educational level identified more risk factors and more preventive actions. In some cases, the magnitude of these inequalities was very significant. However, outcomes in this regard were not entirely unequivocal. For example, women with high education and SES identified physical activity in a very prominent way, whilst women with low education or SES gave more relevance to the consumption of fruit and vegetables, with inequalities disappearing in relation to this response. This could be due to differences in awareness, subjective perceptions of personal risk or existing barriers to the adoption of preventive actions $[5,10,14,35]$.

More than $60 \%$ of men and women considered engaging in physical activity to be the main preventive activity, followed by consumption of fruits and vegetables in women, and smoking cessation in men. Clinical measures were indicated by less than a third of participants, with more women than men opting for such responses. The preventive actions indicated by the present population are in line with scientific evidence and with recommendations included in European guidelines [16,33]. However, stress control is not considered as a relevant preventive action, despite being identified by participants in the present study as the main risk factor for CHD. This may be due to the fact that individuals feel that stress is beyond their personal control as it is associated with adverse 
life conditions. In other words, the strong impact of social health determinants on stress means that social and community strategies are needed to control it [36,37].

The reasons behind women's lower awareness is likely to be multi-factorial [38]. We have already pointed out the misconceptions about epidemiological relevance of cardiovascular disease among women, and that they give greater importance to other health problems. We have also noted the lack of gender-specific strategies to increase awareness. Additionally, results of the present study also show that doctors are less likely to discuss CHD risk with women than with men, possibly due to misperceptions that young women are not at risk [39]. This may be important in determining sex differences in awareness [40] as it deprives women of highly relevant information which could assist them in incorporating positive changes into their lifestyles.

Furthermore, over $80 \%$ of participants, irrespective of gender, agreed that "men who are highly stressed executive professionals" are especially prone to heart attacks. Such a belief exists despite having no basis in any available scientific evidence [36], denoting a clear gender and class bias in relation to CHD risk. On the other hand, the fact that almost 70\% of women knew that the risk of CHD increases with menopause, regardless of age, is a much more positive finding, although small inequalities did emerge according to educational level and SES.

The present study has some strengths and limitations. The study employed a representative sample of the European population. Nonetheless, the European Union is made up of a large number of countries which are highly heterogeneous in relation to key aspects, including cardiovascular health indicators, and the sampling approach used resulted in some countries being over-represented relative to others. Further, only a limited number of countries were considered although criteria used to select the countries from which the sample of participants was drawn were based on the aspects believed to be the most important to reflect. In addition, the approach taken enabled gender differences to be analyzed, unlike in most studies on awareness. This allowed us to examine outcomes in relation to the general population, and to women in particular. Finally, the survey entailed a validated questionnaire which was administered via telephone for feasibility reasons. A methodologically sound approach was taken, employing cutting-edge technology and managed by one of the most assured companies on the continent.

\section{Conclusions}

In conclusion, awareness of CAD is essential for individuals to be able to adopt preventive actions and healthy behaviors, identify main heart attack symptoms and reach emergency services on time. We have shown that the awareness of the European population needs improvement. Campaigns run in other countries have been identified to have produced good outcomes and present findings call for their further implementation [10,41]. More importantly, given the European advantages of having universal health care systems integrated within public health policies [42], a strategy should be developed based on the strong implementation of primary care which includes institutional policy committed to gender equality. This could be key to reducing social and gender inequalities in health [43].

Supplementary Materials: The following are available online at https:/ / www.mdpi.com/article/10 .3390/ijerph19031388/s1, Table S1: Demographic characteristics of respondents to the CHD awareness survey according to age, Table S2: Awareness of selected leading health issues, warning signs of a heart attack and responses to signs of a heart attack, according to age, Table S3: Main risk factors, preventive actions and individuals most at risk in relation to cardiovascular disease, according to age, Table S4: Demographic characteristics of respondents to the CHD awareness survey, according to socioeconomic status, Table S5: Demographic characteristics of respondents to the CHD awareness survey, according to education, Table S6: Prediction of selected indicators of awareness. Figure S1: Frequencies pertaining to selected indicators of awareness. 
Author Contributions: V.R.-Z., A.H.E.M.M., A.W., I.M.-R. and A.D.-C. conceptualize and organized the project. A.D.-C. and I.M.-R. developed the methodology and the instruments for the study. A.D.-C., I.M.-R., V.R.-Z., A.S., A.W., F.R.-O. and F.B. participated in the validation of the questionnaire. A.D.C. and I.M. were responsible for data collection. A.D.-C., I.M.-R. and E.C.K. did the analysis. All authors revised different versions of the manuscript. All authors contributed to the article and approved the submitted version. All authors have read and agreed to the published version of the manuscript.

Funding: Funding was provided by European Commission (DG-SANCO), SANCO/2013/C1/008 17.031800/14/698167; and grant PI19/00561 from the Ministry of Science, Innovation and Universities, Madrid, Spain. Also, Vera Regitz-Zagrosek was also supported by Grant Agreement number: 824585GE Academy-H2020-SwafS-2018-2020/H2020-SwafS-2018-1.

Institutional Review Board Statement: The study was conducted according to the guidelines of the Declaration of Helsinki. The study protocol was approved by the Research Ethics Committee of the Andalusia Regional Health Department School of Public Health (1118N15), Granada, Spain.

Informed Consent Statement: Informed consent was obtained from all subjects involved in the study.

Data Availability Statement: The data used for the study is available from the corresponding author upon request.

Acknowledgments: The authors thank the participants for their collaboration.

Conflicts of Interest: The authors declare no conflict of interest.

\section{References}

1. Timmis, A.; Townsend, N.; Gale, C.P.; Torbica, A.; Lettino, M.; Petersen, S.E.; Mossialos, E.A.; Maggioni, A.P.; Kazakiewicz, D.; May, H.T.; et al. European Society of Cardiology: Cardiovascular Disease Statistics 2019. Eur. Heart J. 2020, 41, 12-85. [CrossRef] [PubMed]

2. Eurostat Cardiovascular Diseases Statistics. Available online: https://ec.europa.eu/eurostat/statistics-explained/index.php? title=Cardiovascular_diseases_statistics (accessed on 11 October 2021).

3. Yusuf, S.; Joseph, P.; Rangarajan, S.; Islam, S.; Mente, A.; Hystad, P.; Brauer, M.; Kutty, V.R.; Gupta, R.; Wielgosz, A.; et al. Modifiable risk factors, cardiovascular disease, and mortality in 155,722 individuals from 21 high-income, middle-income, and low-income countries (PURE): A prospective cohort study. Lancet 2019, 395, 795-808. [CrossRef]

4. National Institute for Health and Care Excellence. NICE Impact: Cardiovascular Disease Prevention. Available online: https:/ / www.nice.org.uk/media/default/about/what-we-do/into-practice/measuring-uptake/nice-impact-cardiovasculardisease-prevention.pdf (accessed on 10 September 2021).

5. $\quad$ Oertelt-Prigione, S.; Seeland, U.; Kendel, F.; Rücke, M.; Flöel, A.; Gaissmaier, W.; Heim, C.; Schnabel, R.; Stangl, V.; RegitzZagrosek, V. Cardiovascular risk factor distribution and subjective risk estimation in urban women-The BEFRI Study: A randomized cross-sectional study. BMC Med. 2015, 13, 1-9. [CrossRef] [PubMed]

6. Thakkar, J.; Heeley, E.; Chalmers, J.; Chow, C.K. Inaccurate risk perceptions contribute to treatment gaps in secondary prevention of cardiovascular disease. Intern. Med. J. 2015, 46, 339-346. [CrossRef] [PubMed]

7. Ramirez, F.D.; Chen, Y.; Di Santo, P.; Simard, T.; Motazedian, P.; Hibbert, B. Association Between Self-Reported Potentially Modifiable Cardiac Risk Factors and Perceived Need to Improve Physical Health: A Population-Based Study. J. Am. Heart Assoc. 2017, 6. [CrossRef]

8. Nehme, Z.; Andrew, E.; Bernard, S.; Patsamanis, H.; Cameron, P.; Bray, J.; Meredith, I.T.; Smith, K. Impact of a public awareness campaign on out-of-hospital cardiac arrest incidence and mortality rates. Eur. Heart J. 2016, 38, 1666-1673. [CrossRef]

9. Mosca, L.; Mochari, H.; Christian, A.; Berra, K.; Taubert, K.; Mills, T.; Burdick, K.A.; Simpson, S.L. National Study of Women's Awareness, Preventive Action, and Barriers to Cardiovascular Health. Circulation 2006, 113, 525-534. [CrossRef]

10. Mosca, L.; Hammond, G.; Greenberger, H.M.; Towfighi, A.; Albert, M.A. Fifteen-Year Trends in Awareness of Heart Disease in Women. Circulation 2013, 127, 1254-1263. [CrossRef]

11. Naylor-Wardle, J.; Rowland, B.; Kunadian, V. Socioeconomic status and cardiovascular health in the COVID-19 pandemic. Heart 2021, 107, 358-365. [CrossRef]

12. Münzel, T.; Miller, M.R.; Sørensen, M.; Lelieveld, J.; Daiber, A.; Rajagopalan, S. Reduction of environmental pollutants for prevention of cardiovascular disease: It's time to act. Eur. Heart J. 2020, 41, 3989-3997. [CrossRef]

13. EUGenMed, T.; Cardiovascular Clinical Study Group; Regitz-Zagrosek, V.; Oertelt-Prigione, S.; Prescott, E.; Franconi, F.; Gerdts, E.; Foryst-Ludwig, A.; Maas, A.H.; Kautzky-Willer, A.; et al. Gender in cardiovascular diseases: Impact on clinical manifestations, management, and outcomes. Eur. Heart J. 2015, 37, 24-34. [CrossRef] 
14. McDonnell, L.A.; Pipe, A.L.; Westcott, C.; Perron, S.; Younger-Lewis, D.; Elias, N.; Nooyen, J.; Reid, R.D. Perceived vs Actual Knowledge and Risk of Heart Disease in Women: Findings From a Canadian Survey on Heart Health Awareness, Attitudes, and Lifestyle. Can. J. Cardiol. 2014, 30, 827-834. [CrossRef]

15. Bolívar Muñoz, J.; Martínez Cassinello, R.; Mateo Rodríguez, I.; Torres Ruiz, J.M.; Pascual Martínez, N.; Rosell-Ortiz, F.; Reina Toral, A.; Martín Castro, C.; Daponte Codina, A. Actuación de los pacientes ante un síndrome coronario agudo: Diferencias desde una perspectiva de género. Emergencias 2013, 25, 23-30.

16. Visseren, F.L.J.; Mach, F.; Smulders, Y.M.; Carballo, D.; Koskinas, K.C.; Bäck, M.; Benetos, A.; Biffi, A.; Boavida, J.-M.; Capodanno, D.; et al. 2021 ESC Guidelines on cardiovascular disease prevention in clinical practice: Developed by the Task Force for cardio-vascular disease prevention in clinical practice with representatives of the European Society of Cardiology and 12 medical societies With the special contribution of the European Association of Preventive Cardiology (EAPC). Eur. Heart J. 2021, 42, 3227-3337. [CrossRef]

17. Erhardt, L.; Hobbs, F.D.R. Public perceptions of cardiovascular risk in five European countries: The react survey. Int. J. Clin. Pr. 2002, 56, 638-644.

18. Cushman, M.; Shay, C.M.; Howard, V.J.; Jiménez, M.C.; Lewey, J.; McSweeney, J.C.; Newby, L.K.; Poudel, R.; Reynolds, H.R.; Rexrode, K.M.; et al. Ten-Year Differences in Women's Awareness Related to Coronary Heart Disease: Results of the 2019 American Heart Association National Survey: A Special Report From the American Heart Association. Circulation 2021, 143, e239-e248. [CrossRef]

19. Mata, J.; Frank, R.; Gigerenzer, G. Symptom recognition of heart attack and stroke in nine European countries: A representative survey. Health Expect. 2012, 17, 376-387. [CrossRef]

20. Mahajan, S.; Valero-Elizondo, J.; Khera, R.; Desai, N.R.; Blankstein, R.; Blaha, M.J.; Virani, S.S.; Kash, B.A.; Zoghbi, W.A.; Krumholz, H.M.; et al. Variation and Disparities in Awareness of Myocardial Infarction Symptoms Among Adults in the United States. JAMA Netw. Open 2019, 2, e1917885. [CrossRef]

21. Intas, G.; Tsolakoglou, J.; Stergiannis, P.; Chalari, E.; Fildissis, G. Do Greek citizens have minimum knowledge about heart attack ? A survey. Health Sci. J. 2015, 9, 1-7.

22. Park, K.S. Factors affecting awareness of myocardial infarction symptoms among the general public. Epidemiology Health 2020, 42, e2020032. [CrossRef]

23. Birnbach, B.; Höpner, J.; Mikolajczyk, R. Cardiac symptom attribution and knowledge of the symptoms of acute myocardial infarction: A systematic review. BMC Cardiovasc. Disord. 2020, 20, 1-12. [CrossRef] [PubMed]

24. Whitaker, S.; Baldwin, T.; Tahir, M.; Choudhry, O.; Senior, A.; Greenfield, S. Public knowledge of the symptoms of myocardial infarction: A street survey in Birmingham, England. Fam. Pract. 2011, 29, 168-173. [CrossRef] [PubMed]

25. Merz, C.N.B.; Shaw, L.J.; Reis, S.; Bittner, V.; Kelsey, S.F.; Olson, M.; Johnson, B.D.; Pepine, C.J.; Mankad, S.; Sharaf, B.L.; et al Insights From the NHLBI-Sponsored Women's Ischemia Syndrome Evaluation (WISE) Study: Part II: Gender Differences in Presentation, Diagnosis, and Outcome With Regard to Gender-Based Pathophysiology of Atherosclerosis and Macrovascular and Microvascular Coronary Disease. J. Am. Coll. Cardiol. 2006, 47, S21-S29. [CrossRef]

26. Van Oosterhout, R.E.M.; De Boer, A.R.; Maas, A.H.E.M.; Rutten, F.H.; Bots, M.L.; Peters, S.A.E. Sex Differences in Symptom Presentation in Acute Coronary Syndromes: A Systematic Review and Meta-analysis. J. Am. Heart Assoc. 2020, 9 , e014733. [CrossRef]

27. Fang, J.; Luncheon, C.; Ayala, C.; Odom, E.; Loustalot, F. Awareness of heart attack symptoms and response among adults-United States, 2008, 2014, and 2017. Morb. Mortal. Wkly. Rep. 2019, 68, 101. [CrossRef]

28. Kopeć, G.; Sobien, B.; Podolec, M.; Dziedzic, H.; Zarzecka, J.; Loster, B.; Pajak, A.; Podolec, P. Knowledge of a patient-dependant phase of acute myocardial infarction in Polish adults: The role of physician's advice. Eur. J. Public Health 2010, 21, 603-608. [CrossRef]

29. Albarqouni, L.; Smenes, K.; Meinertz, T.; Schunkert, H.; Fang, X.; Ronel, J.; Ladwig, K.-H. Patients' knowledge about symptoms and adequate behaviour during acute myocardial infarction and its impact on delay time. Patient Educ. Couns. 2016, 99, 1845-1851. [CrossRef]

30. Garrido, D.; Petrova, D.; Catena, A.; Ramírez-Hernández, J.A.; Garcia-Retamero, R. Recognizing a Heart Attack: Patients' Knowledge of Cardiovascular Risk Factors and Its Relation to Prehospital Decision Delay in Acute Coronary Syndrome. Front. Psychol. 2020, 11, 2056. [CrossRef]

31. Coughlin, S.S.; Young, L. Social Determinants of Myocardial Infarction Risk and Survival: A Systematic Review. Eur. J. Cardiovasc. Res. 2020, 2020, 1-12. [CrossRef]

32. Keller, A.; Litzelman, K.; Wisk, L.E.; Maddox, T.; Cheng, E.R.; Creswell, P.D.; Witt, W.P. Does the perception that stress affects health matter? The association with health and mortality. Health Psychol. 2012, 31, 677-684. [CrossRef]

33. Yusuf, S.; Hawken, S.; Ôunpuu, S.; Dans, T.; Avezum, A.; Lanas, F.; McQueen, M.; Budaj, A.; Pais, P.; Varigos, J.; et al. Effect of potentially modifiable risk factors associated with myocardial infarction in 52 countries (the INTERHEART study): Case-control study. Lancet 2004, 364, 937-952. [CrossRef]

34. Kim, H.-S.; Lee, H.; Kim, K.; Park, H.-K.; Park, K.-S.; Kang, G.W.; Shin, H.-Y.; Kim, R.B.; Oh, G.-J.; Seo, J.H.; et al. The General Public's Awareness of Early Symptoms of and Emergency Responses to Acute Myocardial Infarction and Related Factors in South Korea: A National Public Telephone Survey. J. Epidemiology 2016, 26, 233-241. [CrossRef] 
35. Cainzos-Achirica, M.; Blaha, M.J. Cardiovascular risk perception in women: True unawareness or risk miscalculation? BMC Med. 2015, 13, 1-4. [CrossRef]

36. Fonarow, G.C.; Gawlinski, A. Rationale and design of the Cardiac Hospitalization Atherosclerosis Management Program at the University of California Los Angeles. Am. J. Cardiol. 2000, 85, 10-17. [CrossRef]

37. Havranek, E.P.; Mujahid, M.S.; Barr, D.A.; Blair, I.V.; Cohen, M.S.; Cruz-Flores, S.; Smith, G.D.; Himmelfarb, C.D.; Lauer, M.S.; Lockwood, D.W.; et al. Social Determinants of Risk and Outcomes for Cardiovascular Disease: A Scientific Statement from the American Heart Association. Circulation 2015, 132, 873-898. [CrossRef]

38. Vogel, B.; Acevedo, M.; Appelman, Y.; Merz, C.N.B.; Chieffo, A.; A Figtree, G.; Guerrero, M.; Kunadian, V.; Lam, C.S.P.; Maas, A.H.E.M.; et al. The Lancet women and cardiovascular disease Commission: Reducing the global burden by 2030. Lancet 2021, 397, 2385-2438. [CrossRef]

39. Lichtman, J.H.; Leifheit-Limson, E.C.; Watanabe, E.; Allen, N.B.; Garavalia, B.; Garavalia, L.S.; Spertus, J.A.; Krumholz, H.M.; Curry, L.A. Symptom Recognition and Healthcare Experiences of Young Women With Acute Myocardial Infarction. Circ. Cardiovasc. Qual. Outcomes 2015, 8, S31-S38. [CrossRef]

40. Leifheit-Limson, E.C.; D'Onofrio, G.; Daneshvar, M.; Geda, M.; Bueno, H.; Spertus, J.A.; Krumholz, H.M.; Lichtman, J.H. Sex Differences in Cardiac Risk Factors, Perceived Risk, and Health Care Provider Discussion of Risk and Risk Modification Among Young Patients With Acute Myocardial Infarction. J. Am. Coll. Cardiol. 2015, 66, 1949-1957. [CrossRef]

41. Long, T.; Taubenheim, A.M.; Wayman, J.; Temple, S.; Ruoff, B.A. The Heart Truth: Using the Power of Branding and Social Marketing to Increase Awareness of Heart Disease in Women. Soc. Mark. Q. 2008, 14, 3-29. [CrossRef]

42. Kouvari, M.; Souliotis, K.; Yannakoulia, M.; Panagiotakos, D.B. Cardiovascular Diseases in Women: Policies and Practices around the Globe to Achieve Gender Equity in Cardiac Health. Risk Manag. Healthc Policy. 2020, 13, 2079-2094. [CrossRef]

43. Salmi, L.-R.; Barsanti, S.; Bourgueil, Y.; Daponte, A.; Piznal, E.; Ménival, S.; Leleu, H.; Jusot, F.; Saton, M.-O.; Kovacs, Z.; et al. Interventions addressing health inequalities in European regions: The AIR project. Health Promot. Int. 2015, 32, 430-441. [CrossRef] 